\title{
On the effect of uncertainty on personal vaccination decisions
}

\author{
Christophe Courbage* \\ Geneva School of Business Administration, \\ University of Applied Sciences Western Switzerland (HES-SO), \\ Geneva, Switzerland \\ E-Mail: christophe.courbage@hesge.ch \\ Richard Peter \\ University of Iowa, Department of Finance, \\ Iowa City, Iowa, USA \\ E-Mail: richard-peter@uiowa.edu
}

\begin{abstract}
This paper investigates the effect of ambiguity on personal vaccination decisions. We first characterize the vaccination decision in the absence of ambiguity. We then show that uncertainty about the probability of side effects and the efficacy of the vaccine always reduces take-up under ambiguity aversion. However, uncertainty about the underlying disease, being the probability of sickness or the probability of a severe course of disease, may either encourage or discourage vaccination. Our results are relevant for policy because reducing uncertainty associated with the vaccine always has the desired effect whereas reducing uncertainty associated with the disease may have unintended consequences.
\end{abstract}

Keywords: primary prevention $\cdot$ vaccination $\cdot$ uncertainty $\cdot$ ambiguity

JEL-Classification: D11 $\cdot$ D81 $\cdot$ I12

${ }^{*}$ Corresponding author 


\section{Introduction}

Vaccination is considered as one of the main tools to eradicate pandemics and other infectious diseases (WHO, 2013), calling for a better understanding of individual vaccination decisions. While vaccines reduce the individual's probability of becoming sick, they may entail side effects such as headaches, fevers, muscle and joint aches or even death as illustrated by the Covid-19 pandemic (CDC, 2021). Therefore, at the individual level, the decision to vaccinate implies a trade-off between its costs and benefits (Crainich et al., 2019).

In many circumstances, people take vaccination decisions under uncertainty. For instance, the Covid-19 pandemic has shown that people face limited or contradictory sources of information regarding the disease (de la Oliva et al., 2021). They are uncertain about the chance of being infected, the efficacy of the vaccine, e.g., due to the evolution of new strains, and its side effects. All these factors are likely to affect people's behavior (Han et al., 2018). Since Ellsberg's (1961) famous paradox, it is well known that individuals are often ambiguity-averse, in particular in medical decision-making (Portnoy et al., 2011; Attema et al., 2018). Against this background, we analyze how ambiguity affects individual vaccination decisions.

Our work connects to the literature on self-protection (see Ehrlich and Becker, 1972), which has largely addressed financial risks (Dionne and Eeckhoudt, 1985; Eeckhoudt and Gollier, 2005; Peter, 2021b) and health risks (Courbage and Rey, 2006; Peter, 2021a). Yet, vaccination is a binary choice whereas the classical model of self-protection considers the optimal level of effort, and side effects need to be incorporated. We also extend the decision-threshold framework, introduced by Pauker and Kassirer (1975). Threshold analysis is well-rooted in medical decision-making (Felder and Mayrhofer, 2018), but has hardly addressed preventive decisions (Courbage and Rey, 2016). Finally, we extend Berger et al.'s (2013) study of curative care under ambiguity to preventive care.

We first provide a characterization of the vaccination decision in the absence of ambiguity. We then show that uncertainty about the probability of side effects and the efficacy of the vaccine always reduce take-up of the vaccine under ambiguity aversion. However, uncertainty about the underlying disease itself, being the probability of sickness or the probability of a severe course of disease, may either encourage or discourage vaccination. This is relevant for policy because reducing uncertainty associated with the vaccine always has the desired effects whereas reducing uncertainty associated with the disease may have unintended consequences.

\section{The model}

We consider an individual with a two-argument von Neumann-Morgenstern utility function over consumption and health denoted by $u(C, H) . C$ represents consumption of an aggregate good and $H$ measures health. We assume for simplicity that health can be measured with a single variable. Both consumption and health are valued, $u_{C}>0$ and $u_{H}>0$.

The individual faces a binary risk of sickness. She becomes sick with probability $p \in(0,1)$ and stays healthy with probability $(1-p)$. Sickness lowers health and is accompanied by (uninsured) medical expenditures or lost wages. $C_{i}$ and $H_{i}$ denote consumption and health in the 
healthy state $(i=h)$ and the sick state $(i=s)$, with $C_{s}<C_{h}$ and $H_{s}<H_{h}$. The individual's expected utility is given by

$$
U^{n}=p u\left(C_{s}, H_{s}\right)+(1-p) u\left(C_{h}, H_{h}\right)
$$

where superscript $n$ is shorthand for "not vaccinated."

A vaccine is available, and we model its effects along the lines of Crainich et al. (2019). The vaccine reduces the probability of sickness by $e \in(0, p)$. We assume that the monetary cost of the vaccine is zero either because it is covered by insurance or because it is negligibly small relative to consumption. Vaccination introduces the risk of side effects. There is a probability $q \in(0,1)$ for the individual to experience complications of $c>0$. We assume the risk of sickness and the risk of side effects to be independent. ${ }^{1}$ Expected utility is then given by

$$
\begin{aligned}
U^{v}= & (p-e)\left(q u\left(C_{s}, H_{s}-c\right)+(1-q) u\left(C_{s}, H_{s}\right)\right) \\
& +(1-p+e)\left(q u\left(C_{h}, H_{h}-c\right)+(1-q) u\left(C_{h}, H_{h}\right)\right),
\end{aligned}
$$

where superscript $v$ is shorthand for "vaccinated." $U^{v}$ is increasing in the effectiveness of the vaccine $e$, and decreasing in the probability and severity of side effects $q$ and $c$.

\section{The vaccination decision}

Vaccination is valuable if and only if $U^{v} \geq U^{n}$, so that the vaccine raises expected utility. ${ }^{2} \mathrm{We}$ will distinguish the individual's risk attitude over health, which can be averse, neutral or loving depending on the sign of $u_{H H}$, and her attitude over correlation between consumption and health, which can also be averse, neutral or loving, depending on the sign of $u_{C H}$. Recent empirical evidence on these preference traits is mixed (see, e.g., Attema et al., 2019). The following result characterizes the vaccination decision, see Appendix A for a proof.

\section{Proposition 1.}

(i) Individuals with $u_{H H} \leq 0$ and $u_{C H} \leq 0$ vaccinate if and only if $p \leq p^{*}$. The threshold $p^{*}$ is decreasing in the severity of side effects with $p^{*}=1$ for $c \leq \check{c}$ (always vaccinate) and $p^{*}=e$ for $c \geq \hat{c}$ (never vaccinate). For $c \in(\check{c}, \hat{c}), p^{*}$ is between e and 1 .

(ii) Individuals with $u_{H H} \geq 0$ and $u_{C H} \geq 0$ vaccinate if and only if $p \geq p^{*}$. The threshold $p^{*}$ is increasing in the severity of side effects with $p^{*}=e$ for $c \leq \hat{c}$ (always vaccinate) and $p^{*}=$ 1 for $c \geq \check{c}$ (never vaccinate). For $c \in(\hat{c}, \check{c}), p^{*}$ is between e and 1 .

(iii) For individuals with $u_{H H}<0$ and $u_{C H}>0$ or with $u_{H H}>0$ and $u_{C H}<0$, both cases can occur. The threshold $p^{*}$ is either decreasing or increasing in $c$.

\footnotetext{
${ }^{1}$ Independence implies that individuals who experience side effects are no more or less likely to contract the disease later than those individuals without side effects.

${ }^{2}$ We assume that individuals decide in favor of vaccination when indifferent.
} 
If potential side effects are mild, people will always favor vaccination, and if potential side effects are severe, people will always prefer to remain unvaccinated. In reality, we observe both types of decisions. This is the case for intermediate severity levels of side effects, i.e., for $c \in$ $(\check{c}, \hat{c})$ in case $(i)$ and for $c \in(\hat{c}, \check{c})$ in case (ii). Then, a threshold on the sickness probability separates people in favor from people against vaccination. Notice that the decision rule is the same for anybody whose utility function has the specified derivatives whereas the exact magnitude of $\hat{c}$ and $\check{c}$ depends on preferences and thus on the particular utility function.

Figure 1 represents the two cases outlined in Proposition 1 graphically. ${ }^{3}$ In panel (a), an increase in the severity of side effects lowers $U^{v}$ but increases its slope so that it intersects $U^{n}$ from above. Therefore, the value of vaccination, defined by $V=U^{v}-U^{n}$, is decreasing in $p$ and people vaccinate if and only if $p \leq p^{*}$. In panel (b), an increase in the severity of side effects lowers $U^{v}$ but now decreases its slope so that it intersects $U^{n}$ from below. The value of vaccination is now increasing in $p$ and people vaccinate if and only if $p \geq p^{*}$.

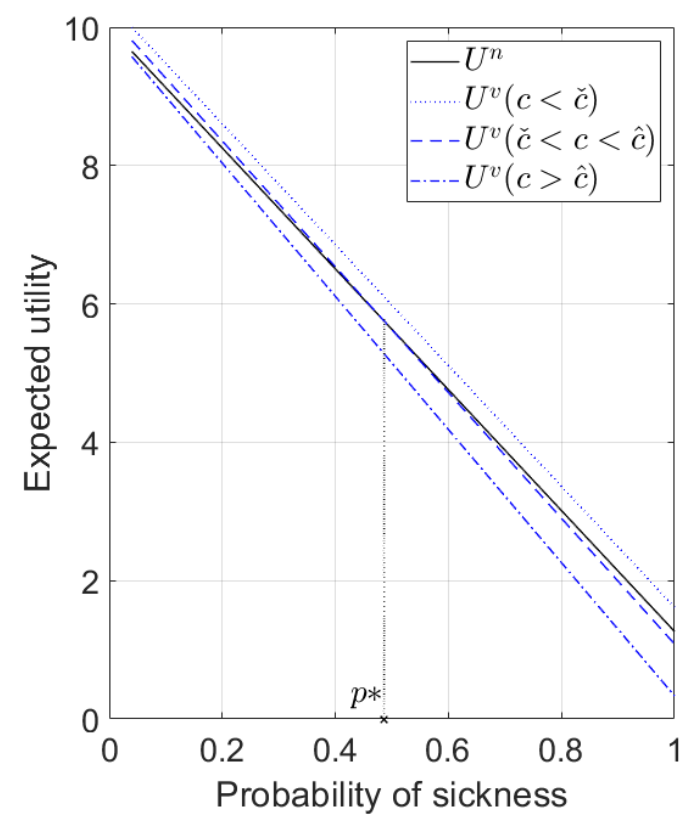

(a) Case (i): $U^{v}$ intersects $U^{n}$ from above

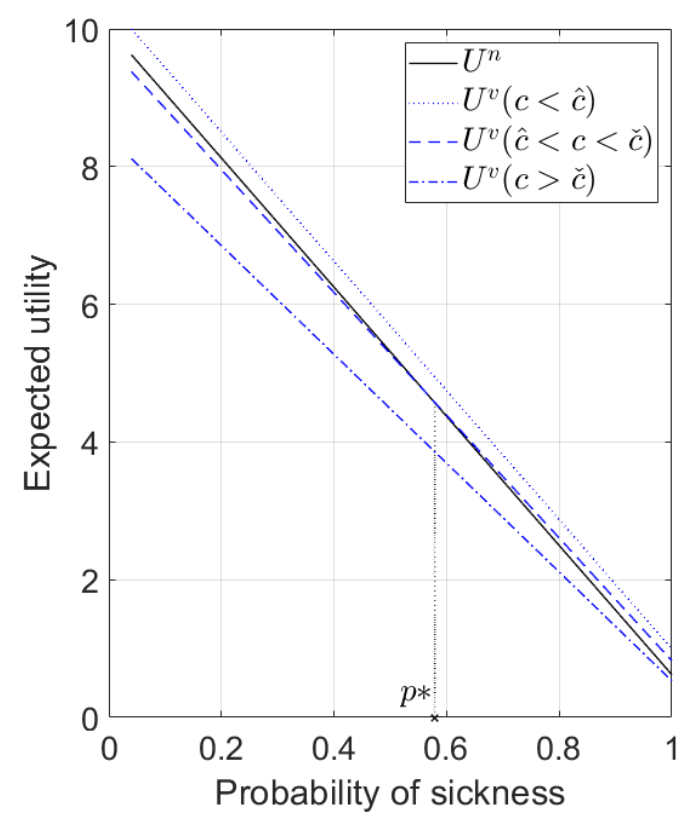

(b) Case (ii): $U^{v}$ intersects $U^{n}$ from below

Figure 1: Illustration of Proposition 1. The blue lines represent expected utility at different levels of side effects. In case $(i)$, for $c \in(\check{c}, \hat{c})$, individuals vaccinate if $p \leq p^{*}$, in case (ii), for $c \in(\hat{c}, \breve{c})$, individuals vaccinate if $p \geq p^{*}$.

Intuition for this distinction can be derived in the spirit of harms disaggregation (see Eeckhoudt et al., 2007). If the individual is health risk- and correlation-averse, she prefers to disaggregate harms and would rather allocate side effects to the healthy state than the sick state,

$$
u\left(C_{h}, H_{h}\right)+u\left(C_{s}, H_{s}-c\right) \leq u\left(C_{h}, H_{h}-c\right)+u\left(C_{s}, H_{s}\right)
$$

\footnotetext{
${ }^{3}$ Appendix B provides the underlying parameter choices and utility functions.
} 
Consequently, side effects increase the loss in utility from sickness. A higher probability of sickness therefore reduces expected utility by more for vaccinated than unvaccinated individuals, which explains the upper bar in Proposition 1(i) and why individuals favor vaccination for a low probability of sickness. If the individual is health risk- and correlation-loving, she prefers to aggregate harms, matters are reversed and individuals favor vaccination for a high probability of sickness.

Proposition 1 can also provide some insights on positive externalities caused by vaccination. As more and more people are vaccinated, the probability of others getting sick is reduced. This will discourage some unvaccinated individuals from getting vaccinated in case (ii) but may surprisingly encourage other unvaccinated individuals to get vaccinated in case (i).

\section{Ambiguity}

We will now investigate how uncertainty affects the individual's vaccination decision. Some sources of uncertainty are associated with the vaccine while others are associated with the disease itself. In the first case, we will consider uncertainty over the probability of side effects and the efficacy of the vaccine. In the second case, we will consider uncertainty over the probability of sickness and the probability of the severity of sickness. This encompasses the main uncertainties currently experienced in the Covid-19 crisis.

We focus on subjective beliefs because people may simply perceive uncertainty, especially when it comes to newly developed vaccines and new pandemics, for personal reasons including lack of information, limited trust in research, the government or the healthcare system, or due to a general sentiment of vaccine skepticism. ${ }^{4}$ However, our model also accommodates objective uncertainty such as scant scientific evidence. Uncertain probabilities are commonly referred to as ambiguity (see Ellsberg, 1961), and recent evidence suggests that people are more pessimistic in medical decisions under ambiguity than under risk, especially for health losses (see Attema et al., 2018). We use Ghirardato et al.'s (2004) $\alpha$-maxmin expected utility model to incorporate ambiguity aversion. In this model, the individual evaluates uncertain prospects via a weighted average over the worst case and the best case. It contains Gilboa and Schmeidler's (1989) famous maxmin model as a special case, which is still one of the most widely-used models of decision-making under ambiguity.

Consider first the probability of side effects, and let priors be built by $\varepsilon$-contamination around $q$ (see Epstein and Wang, 1994). The individual is uncertain about the likelihood of side effects and considers an entire range $Q=[q(1-\varepsilon), \varepsilon+q(1-\varepsilon)]$. People assign a confidence weight of $(1-\varepsilon)$ to $q$, and $\varepsilon$ measures the size of the range. Parameter $\varepsilon$ is commonly interpreted as a measure of perceived ambiguity. Let $\alpha \in[0,1]$ denote the individual's ambiguity aversion. Her perceived welfare from vaccination is now given by

$$
U_{a}^{v}=\alpha \min _{q^{\prime} \in Q} U^{v}+(1-\alpha) \max _{q^{\prime} \in Q} U^{v}
$$

\footnotetext{
${ }_{4}^{4}$ These examples not only lead to increased uncertainty but could also reduce people's subjective belief about the efficacy of vaccination. In this case, our model predicts a lower take-up of the vaccine.
} 
In the case of uncertainty about the efficacy of the vaccine, the individual considers the range of efficacy values $E=[e(1-\varepsilon), \varepsilon+e(1-\varepsilon)]$ with $\varepsilon<(p-e) /(1-e)$ for positive probabilities. Her perceived welfare is derived analogously. The following proposition summarizes the effects of uncertainty associated with the vaccine, see Appendix $\mathrm{C}$ for a proof.

Proposition 2. Under ambiguity aversion, uncertainty about the probability of side effects or the efficacy of the vaccine lowers $p^{*}$ if $u_{H H} \leq 0$ and $u_{C H} \leq 0$, and raises $p^{*}$ if $u_{H H} \geq 0$ and $u_{C H} \geq 0$. For $u_{H H}<0$ and $u_{C H}>0$ or $u_{H H}>0$ and $u_{C H}<0$, both cases are possible.

Uncertainty associated with the vaccine has the intuitive effect that it reduces the perceived value of the vaccine and fewer people vaccinate. What is interesting is that it can be people at high risk or at low risk of contracting the disease who forego the shot, depending on their risk attitude over health and correlation.

Let us now analyze uncertainties associated with the disease and consider the probability of sickness first. Let $P=[p(1-\varepsilon), \varepsilon+p(1-\varepsilon)]$ denote the range of priors with $\varepsilon<(p-e) / p$ to ensure positive probabilities. The following proposition holds, see Appendix D.

Proposition 3. Under ambiguity aversion, uncertainty about the probability of disease always lowers $p^{*}$.

Contrary to Proposition 2, uncertainty about the probability of disease can either discourage or encourage vaccination. If $u_{H H} \leq 0$ and $u_{C H} \leq 0$, a lower $p^{*}$ means that fewer people vaccinate, while if $u_{H H} \geq 0$ and $u_{C H} \geq 0$, a lower $p^{*}$ means that more people vaccinate. If $u_{H H}<0$ and $u_{C H}>0$ or $u_{H H}>0$ and $u_{C H}<0$, both effects are possible.

Finally, we extend the baseline model by allowing for different severity levels of the disease. Let $C_{S S}<C_{s}$ and $H_{S S}<H_{s}$ denote consumption and health if the individual is severely sick as abbreviated by subscript $s s$, and let $\pi$ denote the probability of experiencing a severe course of disease. Let $\Pi=[\pi(1-\varepsilon), \varepsilon+\pi(1-\varepsilon)]$ denote the range of priors with $\varepsilon<(\pi-e) / \pi$. The following proposition holds, see Appendix E.

Proposition 4. Under ambiguity aversion, uncertainty about the probability of a severe course of the disease lowers $p^{*}$ if $u_{H H} \geq 0$ and $u_{C H} \geq 0$; if $u_{H H} \leq 0$ and $u_{C H} \leq 0$, it raises $p^{*}$ if $q$ is below an endogenous threshold $\tilde{q}$ and lowers it otherwise. For $u_{H H}<0$ and $u_{C H}>0$ or $u_{H H}>0$ and $u_{C H}<0$, both cases are possible.

The prevailing case is that uncertainty about the probability of experiencing a severe course of disease encourages vaccination, especially when the probability of side effects is small, but as in the case of uncertainty about the probability of disease, the contrary may occur. 


\section{Conclusion}

In the context of the ongoing Covid-19 pandemic, people across the world have made or must still make a decision to be vaccinated or not in the presence of significant uncertainty. This paper has investigated the effects of ambiguity on individual decisions to vaccinate. It reveals that such effects depend on the source of ambiguity, which has important policy perspectives. Indeed, our results show that reducing uncertainty associated with the vaccine will always encourage vaccination whereas reducing uncertainty associated with the disease may have unintended "side effects" and discourage vaccination for some individuals, depending on their risk preferences over health and correlation.

Various avenues for future research can be considered. First, our model relies on expected utility which has limited descriptive validity, including in the health domain (Bleichrodt et al., 2007). A natural extension would be to consider the rank-dependent utility model (Quiggin, 1982) or reference-dependent models (Kahneman and Tversky, 1979), which have better descriptive validity. Second, the theoretical results of our paper lend themselves to empirical investigations, whether in the form of experimental work or quantitative analysis to investigate the decision to vaccinate under uncertainty. There is no doubt that the current Covid-19 crisis should offer ample opportunities for such follow-up studies. 


\section{References}

Attema, A., Bleichrodt, H. and L'Haridon, O. (2018). Ambiguity preferences for health. Health Economics, 27(11): 1699-1716.

Attema, A., l'Haridon, O. and van de Kuilen, G. (2019). Measuring multivariate risk preferences in the health domain. Journal of Health Economics, $64: 15$-24.

Bleichrodt, H., Abellan-Perpiñan, J.M., Pinto-Prades, J.L., Mendez-Martinez, I. (2007). Resolving inconsistencies in utility measurement under risk: Tests of generalizations of expected utility. Management Science, 53: 469-482

Berger, L., Bleichrodt, H., Eeckhoudt, L. (2013) Treatment decisions under ambiguity. Journal of Health Economics, 32: 559-569.

Centers for Disease Control and Prevention (CDC). (2021). Possible side-effects from vaccines. (Available from: https://www.cdc.gov/vaccines/vac-gen/side-effects.htm) [Accessed on 20 July 2021].

Courbage, C. and Rey, B. (2006). Prudence and optimal prevention for health risks. Health Economics, 15(12): 1323-1327.

Courbage, C. and Rey, B. (2016). Decision thresholds and changes in risk for preventive treatment. Health Economics, 25(1): 111-124.

Crainich, D., Eeckhoudt, L. and Menegatti, M. (2019). Vaccination as a trade-off between risks. Italian Economic Journal, 5: 455-472.

de la Oliva, P., Rodriguez-Rubio, M., García-Rodríguez, J. (2021). Scientific ambiguity in the time of Coronavirus disease 2019. JAMA Pediatrics, 175(3): 318.

Dionne, G. and Eeckhoudt, L. (1985). Self-insurance, self-protection and increased risk aversion. Economics Letters, 17(1-2): 39-42.

Eeckhoudt, L. and Gollier, C. (2005). The impact of prudence on optimal prevention. Economic Theory, 26(4): 989-994.

Eeckhoudt, L., Rey, B. and Schlesinger, H. (2007). A good sign for multivariate risk taking. Management Science, 53(1): 117-124.

Ellsberg, D. (1961). Risk, ambiguity, and the Savage axioms. The Quarterly Journal of Economics, 75(4): 643-669.

Epstein, L.G. and Wang, T. (1994). Intertemporal asset pricing under Knightian uncertainty. Econometrica, 62(2): 283-322.

Ehrlich, I., Becker, G. (1972). Market insurance, self insurance and self protection. Journal of Political Economy, 80: 623-648.

Felder, S. and Mayrhofer, T. (2018). Threshold analysis in the presence of both the diagnostic and the therapeutic risk. The European Journal of Health Economics, 19: 1019-1026.

Ghirardato, P., Maccheroni, F. and Marinacci, M. (2004). Differentiating ambiguity and ambiguity attitude. Journal of Economic Theory, 118(2): 133-173. 
Gilboa, I. und Schmeidler, D. (1989). Maxmin expected utility with non-unique prior. Journal of Mathematical Economics, 18(2): 141-153.

Han, P., Zikmund-Fisher, B., Duarte, C., et al. (2018). Communication of scientific uncertainty about a novel pandemic health threat: Ambiguity aversion and its mechanisms. Journal of Health Communication, 23(5): 435-444.

Kahneman, D. and Tversky, A. (1979). Prospect theory: An analysis of decision under risk. Econometrica, 47(2): 263-292.

Pauker, S. and Kassirer, J. (1975). Therapeutic decision making: a cost benefit analysis. New England Journal of Medicine, 293: 229-234.

Peter, R. (2021a). A fresh look at primary prevention for health risks. Health Economics, 30(5): $1247-1254$

Peter, R. (2021b). Who should exert more effort? Risk aversion, downside risk aversion and optimal prevention. Economic Theory, 71(4): 1259-1281

Portnoy, D., Han, P., Ferrer, R., Klein, W. and Clauser, S. (2011). Physicians' attitudes about communicating and managing scientific uncertainty differ by perceived ambiguity aversion of their patients. Health Expectations, 16: 362-372.

Quiggin, J. (1982). A theory of anticipated utility. Journal of Economic Behavior \& Organization, 3(4): 323-343.

WHO (2013). Global vaccine action plan 2011-2020. World Health Organization, Geneva. 


\section{Appendix A - Proof of Proposition 1}

Both $U^{v}$ and $U^{n}$ are linearly decreasing in $p$. Define the following auxiliary function:

$$
f(c)=q\left[u\left(C_{h}, H_{h}-c\right)-u\left(C_{h}, H_{h}\right)\right]+e\left[u\left(C_{h}, H_{h}\right)-u\left(C_{s}, H_{s}\right)\right] .
$$

$f$ measures the difference between $U^{v}$ and $U^{n}$ when $p \rightarrow e$. It is strictly decreasing in $c$ with $f(0)=e\left[u\left(C_{h}, H_{h}\right)-u\left(C_{s}, H_{s}\right)\right]>0$. Let $\hat{c}$ denote the unique zero of $f$ where it switches from positive to negative. Now define another auxiliary function:

$$
g(c)=e\left[q u\left(C_{h}, H_{h}-c\right)+(1-q) u\left(C_{h}, H_{h}\right)-u\left(C_{s}, H_{s}\right)\right]-(1-e) q\left[u\left(C_{S}, H_{s}\right)-u\left(C_{s}, H_{s}-c\right)\right] .
$$

$g$ measures the difference between $U^{v}$ and $U^{n}$ when $p \rightarrow 1$. It is strictly decreasing in $c$ with $g(0)=e\left[u\left(C_{h}, H_{h}\right)-u\left(C_{s}, H_{s}\right)\right]>0$. Let $\check{c}$ denote the unique zero of $g$ where it switches from positive to negative.

We distinguish between two cases. If $\check{c}<\hat{c}$, then $U^{v}>U^{n}$ for $c \leq \check{c}$ so that vaccination is preferred and $U^{v}<U^{n}$ for $c \geq \hat{c}$ so that remaining unvaccinated is preferred. For $c \in(\check{c}, \hat{c}), U^{v}$ is steeper in $p$ than $U^{n}$ because $U^{v}>U^{n}$ for $p \rightarrow e$ and $U^{v}<U^{n}$ for $p \rightarrow 1$. We can then find a unique $p^{*} \in(e, 1)$ such that $U^{v} \geq U^{n}$ for $p \leq p^{*}$. The threshold $p^{*}$ is decreasing in $c$ because $U^{v}$ is lower the higher $c$ is and $U^{v}$ crosses $U^{n}$ from above. If $\hat{c}<\check{c}$ instead, then $U^{v}>U^{n}$ for $c \leq \hat{c}$ so that vaccination is preferred and $U^{v}<U^{n}$ for $c \geq \check{c}$ so that remaining unvaccinated is preferred. For $c \in(\hat{c}, \check{c}), U^{v}$ is shallower in $p$ than $U^{n}$ because $U^{v}<U^{n}$ for $p \rightarrow e$ and $U^{v}>U^{n}$ for $p \rightarrow 1$. We can then find a unique $p^{*} \in(e, 1)$ such that $U^{v} \geq U^{n}$ for $p \geq p^{*}$. The threshold $p^{*}$ is now increasing in $c$ because $U^{v}$ is lower the higher $c$ is and $U^{v}$ crosses $U^{n}$ from below. ${ }^{5}$

To determine whether $\hat{c}$ or $\check{c}$ is larger, we insert $\hat{c}$ into $g$ and determine the sign. We utilize $f(\hat{c})=0$ and obtain the following:

$$
g(\hat{c})=(1-e) q\left[u\left(C_{h}, H_{h}\right)-u\left(C_{h}, H_{h}-\hat{c}\right)-u\left(C_{s}, H_{s}\right)+u\left(C_{s}, H_{s}-\hat{c}\right)\right] .
$$

We expand the square bracket to

$$
\begin{aligned}
& {\left[u\left(C_{h}, H_{h}\right)-u\left(C_{h}, H_{h}-\hat{c}\right)-u\left(C_{S}, H_{h}\right)+u\left(C_{s}, H_{h}-\hat{c}\right)\right] } \\
+ & {\left[u\left(C_{S}, H_{h}\right)-u\left(C_{S}, H_{h}-\hat{c}\right)-u\left(C_{S}, H_{s}\right)+u\left(C_{s}, H_{s}-\hat{c}\right)\right] . }
\end{aligned}
$$

$u_{H H} \leq 0$ and $u_{C H} \leq 0$ ensure that both terms are non-positive so that $\check{c} \leq \hat{c}$, with a strict inequality if either $u_{H H} \leq 0$ or $u_{C H} \leq 0$ is strict. This shows result (i). $u_{H H} \geq 0$ and $u_{C H} \geq 0$ ensure that both terms are non-negative so that $\check{c} \geq \hat{c}$, with a strict inequality if either $u_{H H} \geq 0$ or $u_{C H} \geq 0$ is strict. This shows result (ii). If $u_{H H}<0$ and $u_{C H}>0$ or if $u_{H H}>0$ and $u_{C H}<0$, we cannot sign $g(\hat{c})$ and both cases are possible, $\check{c}<\hat{c}$ with a threshold $p^{*}$ that is decreasing in $c$, or $\hat{c}<\check{c}$ with a threshold $p^{*}$ that is increasing in $c$. This shows result (iii).

\footnotetext{
${ }^{5}$ The knife-edge case $\check{c}=\hat{c}$ is trivial because either everybody vaccinates for $c \leq \hat{c}$ or nobody vaccinates for $c>\hat{c}$. There are no intermediate scenarios where some individuals vaccinate and others do not.
} 


\section{Appendix B - Parameters underlying Figure 1}

We set $C_{h}=100, C_{s}=50, H_{h}=1$, and $H_{s}=0.65$ for consumption and health in the two states of the world. For the vaccine, we assume $e=0.04$ and $q=0.25$ for the efficacy and probability of side effects. The utility function for panel (a) is $u(C, H)=-C^{-0.5} \cdot H^{-0.5}$, which satisfies $u_{C}>$ $0, u_{H}>0, u_{C C}<0, u_{H H}<0$ and $u_{C H}<0$ so that Proposition $1(i)$ applies. We choose three levels for the severity of side effects, $c=0,0.12$ and 0.24 , and normalize the utility function so that $U^{n}$ and $U^{v}$ are between 0 and 10. For $c=0$, we obtain $U^{v}>U^{n}$, and for $c=0.24$, we obtain $U^{v}<U^{n}$ for all probabilities $p \in(e, 1)$. For $c=0.12$, the vaccination threshold is $p^{*}=0.48$, and people vaccinate if and only if $p \leq p^{*}$.

For panel (b), the utility function is $u(C, H)=\left(-0.5 \cdot C^{-0.5}+0.5\right) \cdot H^{4}$, which satisfies $u_{C}>$ $0, u_{H}>0, u_{C C}<0, u_{H H}>0$ and $u_{C H}>0$ so that Proposition 1(ii) applies. Notice that $u_{H}>0$ and $u_{H H}>0$ require $C \geq 1$, which is given with our choice of parameters. We choose three levels for the severity of side effects, $c=0,0.06$ and 0.24 , and normalize the utility function so that $U^{n}$ and $U^{v}$ are between 0 and 10. For $c=0$, we obtain $U^{v}>U^{n}$, and for $c=0.24$, we obtain $U^{v}<U^{n}$ for all probabilities $p \in(e, 1)$. For $c=0.06$, the vaccination threshold is $p^{*}=0.58$, and people vaccinate if and only if $p \geq p^{*}$.

\section{Appendix C - Proof of Proposition 2}

If $u_{H H} \leq 0$ and $u_{C H} \leq 0$, we know from Proposition 1 that the value of vaccination in the absence of ambiguity, $V=U^{v}-U^{n}$, is decreasing in $p$ with $V\left(p^{*}\right)=0$. If the probability of side effects is uncertain, we obtain

$$
\begin{aligned}
\min _{q^{\prime} \in Q} U^{v}=(p-e) & \left((\varepsilon+q(1-\varepsilon)) u\left(C_{s}, H_{s}-c\right)+(1-q)(1-\varepsilon) u\left(C_{s}, H_{s}\right)\right) \\
+ & (1-p+e)\left((\varepsilon+q(1-\varepsilon)) u\left(C_{h}, H_{h}-c\right)+(1-q)(1-\varepsilon) u\left(C_{h}, H_{h}\right)\right)
\end{aligned}
$$

and

$$
\begin{aligned}
& \max _{q^{\prime} \in Q} U^{v}=(p-e)\left(q(1-\varepsilon) u\left(C_{s}, H_{S}-c\right)+(1-q(1-\varepsilon)) u\left(C_{S}, H_{S}\right)\right) \\
&+(1-p+e)\left(q(1-\varepsilon) u\left(C_{h}, H_{h}-c\right)+(1-q(1-\varepsilon)) u\left(C_{h}, H_{h}\right)\right),
\end{aligned}
$$

and therefore

$$
\begin{aligned}
U_{a}^{v}=(p-e)( & \left.(q+\varepsilon(\alpha-q)) u\left(C_{s}, H_{s}-c\right)+(1-q-\varepsilon(\alpha-q)) u\left(C_{S}, H_{s}\right)\right) \\
& +(1-p+e)\left((q+\varepsilon(\alpha-q)) u\left(C_{h}, H_{h}-c\right)+(1-q-\varepsilon(\alpha-q)) u\left(C_{h}, H_{h}\right)\right) .
\end{aligned}
$$

Ambiguity aversion prevails if $\alpha>q$ because then $U_{a}^{v}<U^{v}$ so that ambiguity makes the individual worse off. The value of vaccination under ambiguity is given by $V_{a}=U_{a}^{v}-U^{n}$ because expected utility when deciding to remain unvaccinated is unaffected by uncertainty over the probability of side effects. Under our assumptions on preferences, $V_{a}$ is also decreasing in $p$, which follows along the lines of the proof of Proposition 1. We then obtain 


$$
V_{a}\left(p^{*}\right)=U_{a}^{v}\left(p^{*}\right)-U^{n}\left(p^{*}\right)<U^{v}\left(p^{*}\right)-U^{n}\left(p^{*}\right)=V\left(p^{*}\right)=0,
$$

so that ambiguity reduces $p^{*}$ and fewer people vaccinate. If $u_{H H} \geq 0$ and $u_{C H} \geq 0$, then $V_{a}$ is increasing in $p$. We obtain $V_{a}\left(p^{*}\right)<0$, which now implies that ambiguity raises $p^{*}$ but again fewer people vaccinate. If $u_{H H}<0$ and $u_{C H}>0$ or if $u_{H H}>0$ and $u_{C H}<0$, then $V_{a}$ can be decreasing or increasing in $p$ and both scenarios are possible. This shows Proposition 2. We omit the proof for uncertainty over the efficacy of the vaccine because it is very similar.

\section{Appendix D - Proof of Proposition 3}

If the probability of disease is uncertain, the individual's expected utility with and without the vaccine are both affected. We obtain

$$
U_{a}^{n}=(p+\varepsilon(\alpha-p)) u\left(C_{s}, H_{s}\right)+(1-p-\varepsilon(\alpha-p)) u\left(C_{h}, H_{h}\right)
$$

for expected utility under ambiguity without the vaccine and

$$
\begin{aligned}
U_{a}^{v}=(p+\varepsilon(\alpha-p)-e)\left(q u\left(C_{s}, H_{s}-c\right)+(1-q) u\left(C_{s}, H_{s}\right)\right) & \\
& +(1-p-\varepsilon(\alpha-p)+e)\left(q u\left(C_{h}, H_{h}-c\right)+(1-q) u\left(C_{h}, H_{h}\right)\right)
\end{aligned}
$$

for expected utility under ambiguity with the vaccine. The value of vaccination under ambiguity, defined as $V_{a}=U_{a}^{v}-U_{a}^{n}$, can be rewritten as

$$
V_{a}=V+\varepsilon(\alpha-p) q \cdot\left[u\left(C_{h}, H_{h}\right)-u\left(C_{h}, H_{h}-c\right)-u\left(C_{s}, H_{s}\right)+u\left(C_{S}, H_{s}-c\right)\right]
$$

where $V=U^{v}-U^{n}$ denotes the value of vaccination without ambiguity.

Then

$$
\frac{\partial V_{a}}{\partial p}=(1-\varepsilon) q \cdot\left[u\left(C_{h}, H_{h}\right)-u\left(C_{h}, H_{h}-c\right)-u\left(C_{s}, H_{s}\right)+u\left(C_{S}, H_{S}-c\right)\right]
$$

If the square bracket is negative, both $V$ and $V_{a}$ are decreasing in $p$, intersect at $p=\alpha$, and $V$ is steeper than $V_{a}$. So if $V$ is uniformly nonnegative, $V_{a}$ is as well and if $V$ is uniformly negative, so is $V_{a}$. The only interesting case is if $V$ changes sign at $p^{*}$. If this occurs in the region where ambiguity aversion prevails (i.e., $\alpha>p^{*}$ ), then $V_{a}\left(p^{*}\right)<0$ and ambiguity lowers $p^{*}$. If the square bracket is positive, both $V$ and $V_{a}$ are increasing in $p$, intersect at $p=\alpha$, and $V$ is steeper than $V_{a}$. Again, the only interesting case is if $V$ changes sign at $p^{*}$. If this occurs in the region where ambiguity aversion prevails (i.e., $\alpha>p^{*}$ ), then $V_{a}\left(p^{*}\right)>0$ and ambiguity lowers $p^{*}$.

\section{Appendix E - Proof of Proposition 4}

In the extended model, we have

$$
U^{n}=p\left[\pi u\left(C_{s S}, H_{s S}\right)+(1-\pi) u\left(C_{s}, H_{s}\right)\right]+(1-p) u\left(C_{h}, H_{h}\right)
$$

for the individual's expected utility without vaccination and 


$$
\begin{aligned}
U^{v}=(p-e) \pi & \left(q u\left(C_{s S}, H_{s S}-c\right)+(1-q) u\left(C_{s S}, H_{s S}\right)\right) \\
& +(p-e)(1-\pi)\left(q u\left(C_{S}, H_{s}-c\right)+(1-q) u\left(C_{s}, H_{s}\right)\right) \\
& +(1-p+e)\left(q u\left(C_{h}, H_{h}-c\right)+(1-q) u\left(C_{h}, H_{h}\right)\right)
\end{aligned}
$$

for the individual's expected utility with the vaccine. $U^{v}$ and $U^{n}$ are linearly decreasing in $p$, and one can use the same approach as in Appendix A to show that Proposition 1 still holds. Uncertainty over the probability of experiencing a severe course of disease results in expected utilities of $U_{a}^{n}$ and $U_{a}^{v}$ with $\pi$ replaced by $\pi+\varepsilon(\alpha-\pi)$ in $U^{v}$ and $U^{n}$. Ambiguity aversion requires $\alpha>\pi$ so that $U_{a}^{n}<U^{n}$ and $U_{a}^{v}<U^{v}$.

Direct computation shows that the value of vaccination under ambiguity is given by

$$
\begin{aligned}
V_{a}=V-\varepsilon(\alpha-\pi) & \\
\cdot & {\left[(p-e) q\left(u\left(C_{S}, H_{s}-c\right)-u\left(C_{S}, H_{s}\right)-u\left(C_{S S}, H_{S S}-c\right)+u\left(C_{s S}, H_{s S}\right)\right)\right.} \\
& \left.-e\left(u\left(C_{S}, H_{s}\right)-u\left(C_{s S}, H_{s S}\right)\right)\right],
\end{aligned}
$$

where $V=U^{v}-U^{n}$ denotes the value of vaccination without ambiguity. The sign of the square bracket determines whether ambiguity increases or reduces the value of vaccination. We always have $u\left(C_{S}, H_{S}\right)>u\left(C_{S S}, H_{S S}\right)$; furthermore, if $u_{H H} \geq 0$ and $u_{C H} \geq 0$, then

$$
u\left(C_{s}, H_{s}-c\right)+u\left(C_{s S}, H_{s S}\right) \leq u\left(C_{S}, H_{s}\right)+u\left(C_{s S}, H_{s S}-c\right),
$$

and the square bracket is negative. As a result, $V_{a}\left(p^{*}\right)>0$, and ambiguity lowers $p^{*}$ because $u_{H H} \geq 0$ and $u_{C H} \geq 0$ ensure that $V_{a}$ is increasing in $p$. If $u_{H H} \leq 0$ and $u_{C H} \leq 0$, the square bracket can be positive or negative. Define

$$
\tilde{q}=\min \left\{\frac{e}{p^{*}-e} \cdot \frac{u\left(C_{S}, H_{S}\right)-u\left(C_{S S}, H_{s S}\right)}{u\left(C_{S}, H_{S}-c\right)-u\left(C_{S}, H_{S}\right)-u\left(C_{S S}, H_{S S}-c\right)+u\left(C_{S S}, H_{S S}\right)}, 1\right\}
$$

then $V_{a}\left(p^{*}\right)>0$ for $q<\tilde{q}$, and ambiguity raises $p^{*}$ because $u_{H H} \leq 0$ and $u_{C H} \leq 0$ ensure that $V_{a}$ is decreasing in $p$. For $q>\tilde{q}$, we obtain $V_{a}\left(p^{*}\right)<0$ and ambiguity lowers $p^{*}$. 